\title{
Porous decellularized tissue engineered hypertrophic cartilage as a scaffold for large bone defect healing
}

\author{
Gráinne M. Cunniffe $e^{\mathrm{a}, \mathrm{b}, \mathrm{c}}$, Tatiana Vinardell ${ }^{\mathrm{d}}$, J. Mary Murphy ${ }^{\mathrm{e}}$, Emmet M. Thompson ${ }^{\mathrm{a}, \mathrm{f}}$, Amos Matsiko ${ }^{\mathrm{a}, \mathrm{f}}$, \\ Fergal J. O’Brien ${ }^{\mathrm{a}, \mathrm{b}, \mathrm{c}, \mathrm{f}}$, Daniel J. Kelly $\mathrm{a}, \mathrm{b}, \mathrm{c}, \mathrm{f}, *$ \\ ${ }^{a}$ Trinity Centre for Bioengineering, Trinity Biomedical Sciences Institute, Trinity College Dublin, Dublin 2, Ireland \\ ${ }^{\mathrm{b}}$ Department of Mechanical and Manufacturing Engineering, School of Engineering, Trinity College Dublin, Dublin 2, Ireland \\ ${ }^{\mathrm{c}}$ Advanced Materials and Bioengineering Research Centre, Trinity College Dublin E' RCSI, Dublin 2, Ireland \\ ${ }^{\mathrm{d}}$ School of Agriculture and Food Science, University College Dublin, Belfield, Dublin 4, Ireland \\ ${ }^{\mathrm{e}}$ REMEDI, National Centre for Biomedical Engineering Science, National University of Ireland Galway, Galway, Ireland \\ ${ }^{\mathrm{f}}$ Tissue Engineering Research Group, Dept. of Anatomy, Royal College of Surgeons in Ireland, Dublin 2, Ireland
}

\section{A R T I C L E I N F O}

\section{Article history:}

Received 20 February 2015

Received in revised form 28 April 2015

Accepted 26 May 2015

Available online $\mathrm{xxxx}$

\section{Keywords:}

Hypertrophic cartilage

Decellularized scaffold

Extracellular matrix

Endochondral ossification

Large bone defect

\begin{abstract}
A B S T R A C T
Clinical translation of tissue engineered therapeutics is hampered by the significant logistical and regulatory challenges associated with such products, prompting increased interest in the use of decellularized extracellular matrix (ECM) to enhance endogenous regeneration. Most bones develop and heal by endochondral ossification, the replacement of a hypertrophic cartilaginous intermediary with bone. The hypothesis of this study is that a porous scaffold derived from decellularized tissue engineered hypertrophic cartilage will retain the necessary signals to instruct host cells to accelerate endogenous bone regeneration. Cartilage tissue (CT) and hypertrophic cartilage tissue (HT) were engineered using human bone marrow derived mesenchymal stem cells, decellularized and the remaining ECM was freeze-dried to generate porous scaffolds. When implanted subcutaneously in nude mice, only the decellularized HT-derived scaffolds were found to induce vascularization and de novo mineral accumulation. Furthermore, when implanted into critically-sized femoral defects, full bridging was observed in half of the defects treated with HT scaffolds, while no evidence of such bridging was found in empty controls. Host cells which had migrated throughout the scaffold were capable of producing new bone tissue, in contrast to fibrous tissue formation within empty controls. These results demonstrate the capacity of decellularized engineered tissues as 'off-the-shelf' implants to promote tissue regeneration.
\end{abstract}

(c) 2015 Acta Materialia Inc. Published by Elsevier Ltd. All rights reserved.

\section{Introduction}

The regeneration of large bone defects through tissue engineering approaches has typically attempted to induce direct osteogenic healing by recapitulating the process of intramembranous ossification. A potential drawback with this approach is that mineralized tissue initially deposited around the periphery of the engineered graft can prevent host vascularization of the implant, leading to the establishment of a necrotic core [1]. In the developing embryo however, most bones form via the process of endochondral ossification, the progressive replacement of a hypertrophic cartilaginous template with bone. During endochondral ossification MSCs condense, differentiate down the chondrogenic lineage and become

\footnotetext{
* Corresponding author at: Department of Mechanical and Manufacturing Engineering, School of Engineering, Trinity College Dublin, Dublin 2, Ireland. Tel. +353 18963947.

E-mail address: kellyd9@tcd.ie (D.J. Kelly).
}

hypertrophic [2]. These hypertrophic chondrocytes secrete biomolecules such as vascular endothelial growth factor (VEGF), which are known to promote angiogenesis, and thus actively induce ingrowth of vasculature as the surrounding matrix is calcifying, in addition to promoting matrix remodelling [3-5]. Hence, it has been suggested that strategies attempting to recapitulate the process of endochondral ossification may provide a superior approach for healing large bone defects [6-10].

A number of studies have demonstrated the capacity of in vitro tissue engineered cartilage to undergo endochondral ossification and generate bone tissue in vivo [11-14]. For example, cartilaginous tissues engineered using human bone marrow derived mesenchymal stem cells (MSCs) have been shown to act as a template for host cell-derived bone formation following subcutaneous implantation into nude mice [15]. Tissue engineering such an autologous cartilaginous graft for clinical applications would involve isolating and expanding the patient's cells, forming a 
construct of suitable dimensions to fit the original defect, culturing this construct for a number of additional weeks in vitro and finally implanting it into the body. There are a number of challenges that would need to be overcome before clinical translation of such a cell-based therapy, including the high cost, complexity, need for two surgical procedures and associated regulatory hurdles [16]. Additionally, with large bone defect healing, increasing graft size can become a major issue, with new bone formation occurring primarily around the periphery of the implant, leading to the search for a suitable osteoinductive material to amplify the ossification process throughout the regenerating tissue. Furthermore, 'off-the-shelf' availability may well determine the extent to which any new therapy achieves widespread clinical adaption. This has led to increased interest in the use of decellularized extracellular matrix (ECM) derived scaffolds for the regeneration of different tissue types [17-22], based on the premise that such biomaterials contain structural and functional molecules to facilitate tissue regeneration. The ECM can be harvested from native tissue or from cell-derived matrix deposited during in vitro culture [23-28]. In the context of endochondral bone regeneration, it remains unclear as to whether decellularized hypertrophic cartilage would retain its pro-angiogenic ability and thus its capacity to direct host-mediated orthotopic bone regeneration.

The hypothesis of this study is that a porous scaffold derived from decellularized hypertrophic cartilage engineered using allogeneic bone marrow derived MSCs will be highly osteoinductive, support vascularization, and will promote bone formation by host progenitor cells. To test this hypothesis, porous scaffolds derived from either engineered cartilage tissues (CT) or engineered hypertrophic cartilage tissues (HT) were implanted subcutaneously into nude mice to evaluate their potential to promote vascularization and ectopic bone formation. The capacity of these HT scaffolds to promote bone regeneration within critically-sized rat femoral defects was then evaluated. The data presented in this study suggest that engineered HT derived scaffolds could be used to improve the repair of critically-sized bone defects.

\section{Experimental section}

\subsection{Experimental design}

This study consisted of two parts. The first experiment initially established the feasibility of generating porous scaffolds entirely from cell-derived ECM, and then compared the ability of scaffolds generated from either engineered cartilage tissue (CT) or engineered hypertrophic cartilage tissue (HT) to promote vascularization and induce ectopic bone formation in a subcutaneous nude mouse model. CT and HT was engineered through self-assembly of human bone marrow-derived MSCs (see specific details below), from which acellular CT and HT scaffolds were generated and implanted into nude mice. Viable tissue engineered grafts were also implanted as positive controls. The second experiment then evaluated the optimum scaffold type from the subcutaneous study to promote bone regeneration in a critically-sized rat femoral defect. Again, this scaffold was implanted acellularly and the study investigated the ability of host cells to infiltrate the structure and induce bone repair.

\subsection{Generating scaffolds for subcutaneous implantation}

Adult human bone marrow-derived stem cells (hMSCs) were expanded to passage 2 and seeded into $6.5 \mathrm{~mm}$ diameter transwell inserts $(3.0 \mu \mathrm{m}$ pore polycarbonate membrane, Corning) at a density of $4 \times 10^{\wedge} 6$ cells per insert [29,30]. Constructs were cultured in either chondrogenic medium (consisting of hgDMEM GlutaMAX supplemented with $100 \mathrm{U} / \mathrm{mL}$ penicillin/streptomycin (both Gibco), $\quad 100 \mu \mathrm{g} / \mathrm{mL}$ sodium pyruvate, $40 \mu \mathrm{g} / \mathrm{mL}$ L-proline, $50 \mu \mathrm{g} / \mathrm{mL}$ L-ascorbic acid-2-phosphate, $4.7 \mu \mathrm{g} / \mathrm{mL}$ linoleic acid, $1.5 \mathrm{mg} / \mathrm{mL}$ bovine serum albumin, $1 \times$ insulin-transferrin-sele nium, $100 \mathrm{nM}$ dexamethasone (all from Sigma-Aldrich), $2.5 \mu \mathrm{g} / \mathrm{mL}$ amphotericin $\mathrm{B}$, and $10 \mathrm{ng} / \mathrm{mL}$ of human transforming growth factor- $\beta 3$ (TGF- $\beta 3$; Prospec-Tany TechnoGene Ltd)) for 6 weeks at $5 \% \mathrm{O}_{2}$ or cultured in chondrogenic medium for the first 4 weeks and switched to hypertrophic medium (consisting of hgDMEM GlutaMAX supplemented with $100 \mathrm{U} / \mathrm{mL}$ penicillin/streptomycin, $100 \mu \mathrm{g} / \mathrm{mL}$ sodium pyruvate, $40 \mu \mathrm{g} / \mathrm{mL}$ L-proline, $50 \mu \mathrm{g} / \mathrm{mL}$ L-ascorbic acid-2-phosphate, $4.7 \mu \mathrm{g} / \mathrm{mL}$ linoleic acid, $1.5 \mathrm{mg} / \mathrm{mL}$ bovine serum albumin, $1 \times$ insulin-transferrin-se lenium, $1 \mathrm{nM}$ dexamethasone, $2.5 \mu \mathrm{g} / \mathrm{mL}$ amphotericin $\mathrm{B}, 1 \mathrm{nM}$ L-thyroxine, and $10 \mathrm{mM} \quad \beta$-Glycerophosphate (both SigmaAldrich)) at $20 \% \mathrm{O}_{2}$ for the final 2 weeks to induce deposition of a hypertrophic cartilage ECM (Fig. 1A). Both cartilage tissue (CT) and hypertrophic cartilage tissue (HT) constructs were then collected directly after in vitro culture and homogenized (IKAT10, IKA Works Inc., NC, USA) to form separate CT and HT slurries (500 mg/ml) in distilled $\mathrm{H}_{2} \mathrm{O}$, placed in custom designed polydimethylsiloxane (PDMS) cylindrical moulds (wells measuring $5 \mathrm{~mm}$ diameter, $3 \mathrm{~mm}$ height) and freeze-dried to create porous $5 \mathrm{~mm} \times 3 \mathrm{~mm}$ constructs (Fig. 2A). Lyophilization was carried out in a freeze-dryer (Triad, Labconco) by bringing the samples to a final freezing temperature of $-30^{\circ} \mathrm{C}$ at a ramp speed of $1{ }^{\circ} \mathrm{C} \mathrm{min}^{-1}$, with a holding step at $-30^{\circ} \mathrm{C}$ for $1 \mathrm{~h}$ to allow for ice-crystal nucleation and growth [25]. The samples were then brought to a drying temperature of $-10^{\circ} \mathrm{C}$ at the same ramp speed and maintained under vacuum ( 0.1 mbarr) for $24 \mathrm{~h}$ to ensure full drying was achieved. No chemical decellularization techniques were applied to these constructs prior to lyophilization, as these scaffolds were implanted into immunocompromised mice. All scaffolds were cross-linked and sterilized using dehydrothermal (DHT; $24 \mathrm{~h}$ at $105^{\circ} \mathrm{C}$ ) and 1-Ethyl-3-(3-dimethylaminopropyl)carbodiim ide (EDAC; $6 \mathrm{mM}$ for $2 \mathrm{~h}$ with two washes in sterile phosphate buffered saline $2 \times 1 \mathrm{~h}$ ) treatments prior to implantation [31]. The hMSCs in this study were used with approval from the National University of Ireland, Galway and University College Hospital ethics committees and after informed consent.

\subsection{Generating scaffolds for orthotopic rat femoral defect study}

Only HT constructs were used to generate scaffolds for this defect study, based on results from the initial nude mouse study. HT grafts were cultured in vitro as before, using hMSCs in a scaffold-free system to deposit hypertrophic cartilage matrix. The resulting blended HT slurry was then decellularized using detergent $(0.1 \%$ SDS, $1 \mathrm{~h})$ and enzymatic $(10 \mathrm{U} / \mathrm{ml}$ DNase, $10 \mathrm{U} / \mathrm{ml}$ RNase, $1 \mathrm{~h}$ ) treatments with washes, before freeze-drying in custom designed polydimethylsiloxane (PDMS) cylindrical moulds (wells measuring $4 \mathrm{~mm}$ diameter, $5 \mathrm{~mm}$ height) to yield porous decellularized scaffolds $(4 \mathrm{~mm} \times 5 \mathrm{~mm})$ for implantation into a rat femoral defect. This decellularization protocol was included in order to remove DNA content while retaining ECM components, and hence reduce the risk of eliciting an immune reaction following implantation. Constructs were freeze-dried, cross-linked and sterilized as described in Section 2.2.

\subsection{Scanning Electron Microscopy (SEM)}

Scaffolds were imaged using SEM to visualize the internal porous structure of the freeze-dried constructs. Imaging was carried out following DHT treatment by fixing constructs to an adhesive carbon stub, sputter coating with gold, and imaging using a lens 
detector with a $5 \mathrm{kV}$ acceleration voltage at calibrated magnifications (Tescan Mira XMU, Tescan USA Inc.).

\subsection{Live/dead confocal microscopy}

Expanded hMSCs (passage 2) were seeded on the CT and HT scaffolds to ensure cells were capable of migrating throughout the construct and that a homogenous distribution could be achieved. Cell viability was assessed after $24 \mathrm{~h}$ using a LIVE/DEAD ${ }^{\circledR} \quad$ viability/cytotoxicity assay kit (Invitrogen, Bio-science, Ireland). Briefly, constructs were cut in half, washed in PBS followed by incubation in PBS containing $2 \mu \mathrm{M}$ calcein AM (green fluorescence of membrane for live cells) and $4 \mu \mathrm{M}$ ethidium homodimer-1 (red fluorescence of DNA for dead cells; both from Cambridge Bioscience, UK). Sections were again washed in PBS, imaged at magnification $\times 10$ with an Olympus FV-1000 Point-Scanning Confocal Microscope (Southend-on-Sea, UK) at 515 and $615 \mathrm{~nm}$ channels and analysed using FV10-ASW 2.0 Viewer software. Z-stack images of 30 scans every $10 \mu \mathrm{m}$ in the cross-section of the scaffold were acquired for a total of $300 \mu \mathrm{m}$ depth.

\subsection{Histology and immunohistochemistry}

Tissue engineered constructs (CT and HT) were stained to examine pre-implantation matrix accumulation following in vitro culture, and repair tissue post-scaffold implantation. Pre-implantation samples were fixed in $4 \%$ paraformaldehyde overnight, dehydrated in a graded series of ethanol and xylenes, and embedded in paraffin wax. In vivo samples were decalcified following fixation using an EDTA treatment (15\% EDTA at pH 7.4) to remove the mineral component prior to embedding. Sections of $8 \mu \mathrm{m}$ were obtained with a microtome (Leica RM2125RT, Ashbourne, Ireland) and affixed to microscope slides. Prior to staining, sections were dewaxed and rehydrated in 100-70\% ethanol followed by distilled water. Cellular infiltration and bone matrix deposition was stained with haematoxylin and eosin (H\&E), whereas glycosaminoglycan and calcium accumulation were analysed histologically using $1 \%$ Alcian Blue in $0.1 \mathrm{M} \mathrm{HCl}$ and $1 \%$ Alizarin Red stains, respectively (all Sigma-Aldrich).

Collagen type $\mathrm{X}$ and VEGF deposition was evaluated using a standard immunohistochemical technique. Briefly, sections ( $n=4$ sections per group) were treated with hydrogen peroxidase (Sigma-Aldrich), followed by enzymatic treatment with Pronase $(0.5 \mathrm{mg} / \mathrm{ml}$, Merck Millipore, Cork, Ireland) in a humidified environment for $5 \mathrm{~min}$ at $37^{\circ} \mathrm{C}$ to enhance the permeability of the extracellular matrix. Sections were incubated with goat serum to block non-specific sites and mouse monoclonal anti-collagen type $\mathrm{X}$ (ab49945, 1:200) and rabbit polyclonal anti-VEGF (ab46154, $1: 200)$ primary antibodies (Abcam, Cambridge, UK) were applied overnight at $4{ }^{\circ} \mathrm{C}$. Next the secondary antibody (goat anti-mouse, $1: 200$, B7151 Sigma-Aldrich, and goat anti-rabbit, 1:200, ab97051 Abcam) were added for $1 \mathrm{~h}$, followed by incubation with $A B C$ reagent (Vectastain PK-400, Vector Labs, Peterborough, UK) for $45 \mathrm{~min}$. Finally, sections were developed with DAB peroxidase (Vector Labs) for $5 \mathrm{~min}$. Positive and negative controls were included in the immunohistochemistry staining protocol for each batch.

\subsection{In vivo subcutaneous implantation}

Four different groups (described below) were implanted ( $n=9$ constructs/scaffolds per group) into transgenic Balb/C nude mice (Harlan, UK) as previously described [32]. These constructs were implanted into subcutaneous pockets created along the central line of the spine, 3 constructs were implanted at the shoulders and 3 constructs at the hips level, (i.e. a total of 6 constructs per mouse). Two experimental groups consisting of devitalized CT and HT scaffolds respectively were implanted acellularly. In addition, two positive control groups consisting of viable cartilage and hypertrophic cartilage tissues (i.e. viable CT and HT grafts) engineered using hMSCs were also implanted. All 4 groups (2 acellular scaffolds and 2 viable tissue engineered grafts) were harvested after 6 weeks in vivo, and tissues were analysed based on their gross appearance, histological, immunohistochemical and biochemical properties. Mice were anaesthetized using an intraperitoneal injection of xylazine hydrochloride and ketamine hydrochloride, Carprofen was added to water for $24 \mathrm{~h}$ post-surgery, and mice were sacrificed by $\mathrm{CO}_{2}$ inhalation. This protocol and study was reviewed and approved by the ethics committee of Trinity College Dublin, Ireland.

\subsection{Femoral defect implantation}

Critically-sized $(5 \mathrm{~mm})$ partial weight-bearing segmental femoral defects were created in immune-competent adult Fischer rats following an established procedure [33]. HT scaffolds were press-fit into the defect site, and repair tissue was harvested for analysis at 4 and 8 weeks post-implantation. One defect was created per animal and $n=4$ scaffolds were implanted per time point, i.e. 8 rats were treated with HT scaffolds and 8 rats contained untreated controls. This animal procedure and study was approved by the ethics committee in the Royal College of Surgeons in Ireland. Briefly, anaesthesia was induced by intraperitoneal injection of xylazine hydrochloride and ketamine hydrochloride, and sedated Fischer rats were maintained by isoflurane-oxygen throughout the surgery. The shaft of the right femur was exposed by dissections and the periosteum was scraped back to allow access to the bone. A weight-bearing polyetheretherketone (PEEK) internal fixation plate was secured to the exposed femur with four screws into pre-drilled holes. A 5-mm mid-diaphyseal defect was then created using a dental drill fitted with 2 small circular parallel saw blades welded to a narrow straight rod separated with a $5 \mathrm{~mm}$ spacer. The defect site was thoroughly irrigated with saline to remove bone debris before it was treated with the HT scaffold. The wounds were closed with sutures and the rats were given atipamezole hydrochloride to reverse sedation. Pain relief was administered via carprofen and buprenorphine subcutaneous injections over the following 2 days. On dates of scheduled explant retrieval, rats were sacrificed by $\mathrm{CO}_{2}$ asphyxiation and cervical dislocation. The repaired femur, with the PEEK plate fixator intact, was carefully separated from the adjacent hip and knee joints for analysis.

\subsection{Micro-computed tomography}

Micro-computed tomography $(\mu \mathrm{CT})$ scans were carried out on the explants from the in vivo subcutaneous and femoral defect studies in order to visualize and quantify mineral content. A Scanco Medical $40 \mu \mathrm{CT}$ system (Scanco Medical, Bassersdorf, Switzerland) was used for evaluation with a $70 \mathrm{kVp}$ X-ray source at $114 \mu \mathrm{A}$. Four constructs were analysed per experimental group. Reconstructed 3D images were generated from the scans and used to visualize mineral distribution throughout the implanted scaffold or control group. Quantification was performed in the subcutaneous study by taking the bone volume readings at a threshold of 165. Quantification was performed in the femoral defect study by setting a threshold of 210 (corresponding to a density of $399.5 \mathrm{mg}$ hydroxyapatite $/ \mathrm{cm}^{3}$ ) and recording the bone volume (BV) within the defect per total volume (BV/TV), using a consistent total volume (central 50 slices of defect) from sample to sample which eliminated original bone from the calculations. 


\subsection{Statistical analysis}

All statistical analyses were carried out using GraphPad. The results are reported as means \pm standard deviation. Groups were analysed by a general linear model for analysis of variance with groups of factors. Tukey's test was used to compare conditions. Significance was accepted at a level of $p<0.05$.

\section{Results}

\subsection{Porous scaffolds can be generated from the ECM of tissue engineered cartilage}

The composition of the ECM generated by MSCs in scaffold-free conditions differed depending on whether they were maintained in chondrogenic or hypertrophic culture media (see Fig. 1A for details of culture conditions). MSCs maintained in chondrogenic medium for 6 weeks, and those switched to hypertrophic medium after 4 weeks of culture, generated tissues that stained positive for sulphated glycosaminoglycans, indicating robust chondrogenesis had occurred in both culture conditions (Fig. 1B). More dramatic differences in the composition of the engineered CT and HT constructs were observed when markers of chondrocyte hypertrophy and endochondral ossification were assessed. Specifically, evidence of calcium and collagen type X deposition was observed in HT constructs, with only weak staining for these hypertrophic markers observed in CT constructs. Furthermore, stronger staining for VEGF accumulation was observed in the HT constructs. This presence of calcific deposits, collagen type $\mathrm{X}$ and VEGF indicates that the engineered HT templates have begun to undergo hypertrophy, and furthermore demonstrates that scaffolds fabricated from CT and HT will contain a unique set of structural and functional cues that may have a differential capacity to promote bone regeneration.

Porous scaffolds were then successfully generated from the ECM of both CT and HT tissue engineered grafts (see Fig. 2A for details of scaffold fabrication), with SEM demonstrating the development of an open interconnected porous structure using a controlled freeze-drying procedure (Fig. $2 \mathrm{~B}$ and $\mathrm{C}$ ). Confocal images of MSCs seeded onto the devitalized scaffolds confirmed that the cells remained viable and could consistently infiltrate into the centre of the biomaterial after $24 \mathrm{~h}$ of culture (Fig. 2D).

\subsection{Scaffolds generated from engineered hypertrophic cartilage ECM promote vascularization and induce mineralization following subcutaneous implantation}

Scaffolds derived from devitalized engineered CT and HT were implanted acellularly into subcutaneous pockets in nude mice, along with positive control vital engineered CT and HT grafts. Macroscopically the two scaffold types appeared different after 6 weeks in vivo (Fig. 3A). The devitalized HT scaffolds were stiff and appeared calcified, with host vasculature penetrating throughout the constructs. In contrast, the devitalized CT scaffolds were soft and more fibrous tissue-like in appearance, with no evidence of blood vessels penetrating into the construct. Both vital CT and vital HT groups were vascularized, with evidence of dense mineralization within both constructs.

The $\mu \mathrm{CT}$ reconstructed images displayed a significant difference in the quantity of mineralization achieved in vivo, with widespread mineral deposition detected consistently throughout the HT scaffolds, compared to minimal deposition occurring in the CT scaffolds (Fig. 3B). The positive control vital CT and vital HT groups also underwent significant mineralization in vivo, generating similar levels of a more bone-like tissue. Significantly higher levels of mineralization were detected in vital CT and HT implants compared to either devitalized scaffold type (Bone Volume values: Vital CT $2.59 \pm 0.46 \mathrm{~mm}^{3}$, Vital HT $1.48 \pm 0.99 \mathrm{~mm}^{3}$, CT scaffold $0.005 \pm 0.00 \mathrm{~mm}^{3}$, HT scaffold $0.12 \pm 0.06 \mathrm{~mm}^{3}$ ). (Note: no evidence of mineral deposition was observed within the viable engineered grafts or the devitalized scaffolds at this $\mu \mathrm{CT}$ threshold level prior to implantation).

Analysis of H\&E stained sections after 6 weeks in vivo revealed evidence of significant blood cell infiltration and some early de novo bone formation in the HT scaffolds, in contrast to the CT scaffold, despite the presence of migrating host cells in both groups (Fig. 4). HT scaffolds also stained more intensely than their CT scaffold counterparts for sGAG, collagen type X and VEGF deposition after 6 weeks in vivo.

\subsection{Scaffolds generated from hypertrophic cartilage ECM can induce} bone healing in critically-sized orthotopic defects

Having demonstrated the capacity of the HT scaffolds to promote vascularization and immature bone formation ectopically, we next undertook a study to determine the capacity of this acellular construct to promote bone regeneration within critically-sized rat femoral defects. $\mu \mathrm{CT}$ analysis revealed full bridging across the defect in HT scaffold treated groups as early as 4 weeks post-implantation, while none of the empty controls had bridged 8 weeks after the creation of the defects (Fig. 5A and B). Full bridging was observed in half of the scaffold treated defects after 8 weeks. Significant animal-to-animal variability in scaffold-mediated bone regeneration was observed, with no significant difference in bone volume per total volume observed after either 4 (empty $-0.015 \pm 0.017$; HT scaffold $-0.063 \pm 0.126 ; p=0.23$ ) or 8 weeks (empty $0.068 \pm 0.05$; HT scaffold $-0.301 \pm 0.355 ; p=0.12$ ) (Fig. $5 \mathrm{C}$ ).

Histological analysis of the healing defects revealed the development of a fibrous tissue within the empty control groups at both 4 and 8 weeks. Repair tissue within the HT scaffold treated group however showed evidence of vascular invasion, scaffold remodelling and bone deposition at 4 weeks and dense mature bone development at 8 weeks, with evidence of more lamellar-like bone forming by this time-point (Fig. 6).

\section{Discussion}

Decellularization of tissue engineered grafts represents a promising strategy to accelerate the translation of such therapies into the clinic by providing 'off-the-shelf' constructs that can facilitate endogenous tissue or organ regeneration [34-36]. The results of this study provide support for the hypothesis that a porous scaffold generated from hypertrophic cartilage ECM, engineered using allogeneic MSCs, will support vascularization and host-mediated bone formation upon implantation. Only the HT-derived scaffold was capable of promoting vascularization and mineralization in an ectopic environment, demonstrating the importance of hypertrophic priming for generating an osteoinductive ECM. In the second phase of the study, it was further demonstrated that this HT derived scaffold could also accelerate regeneration of large bone defects created in the femurs of rats. Together these findings suggest that porous scaffolds derived from decellularized tissue engineered hypertrophic cartilage could be used as 'off-the-shelf' biomaterials for bone regeneration.

The composition of cartilage tissues engineered in either chondrogenic or hypertrophic culture conditions were distinctly different, with HT tissues staining more strongly for calcium and type $\mathrm{X}$ collagen deposition. This is in agreement with previous studies which have demonstrated that early hypertrophic cartilaginous tissues engineered using bone marrow-derived MSCs stained 
(A)

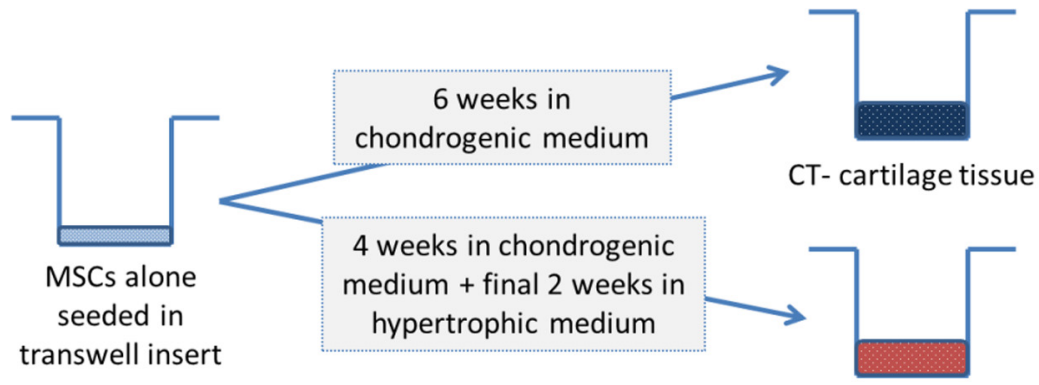

(B)

HT- hypertrophic cartilage tissue

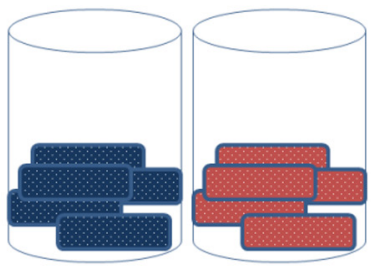

$\mathrm{CT} / \mathrm{HT}$ constructs harvested after 6 weeks
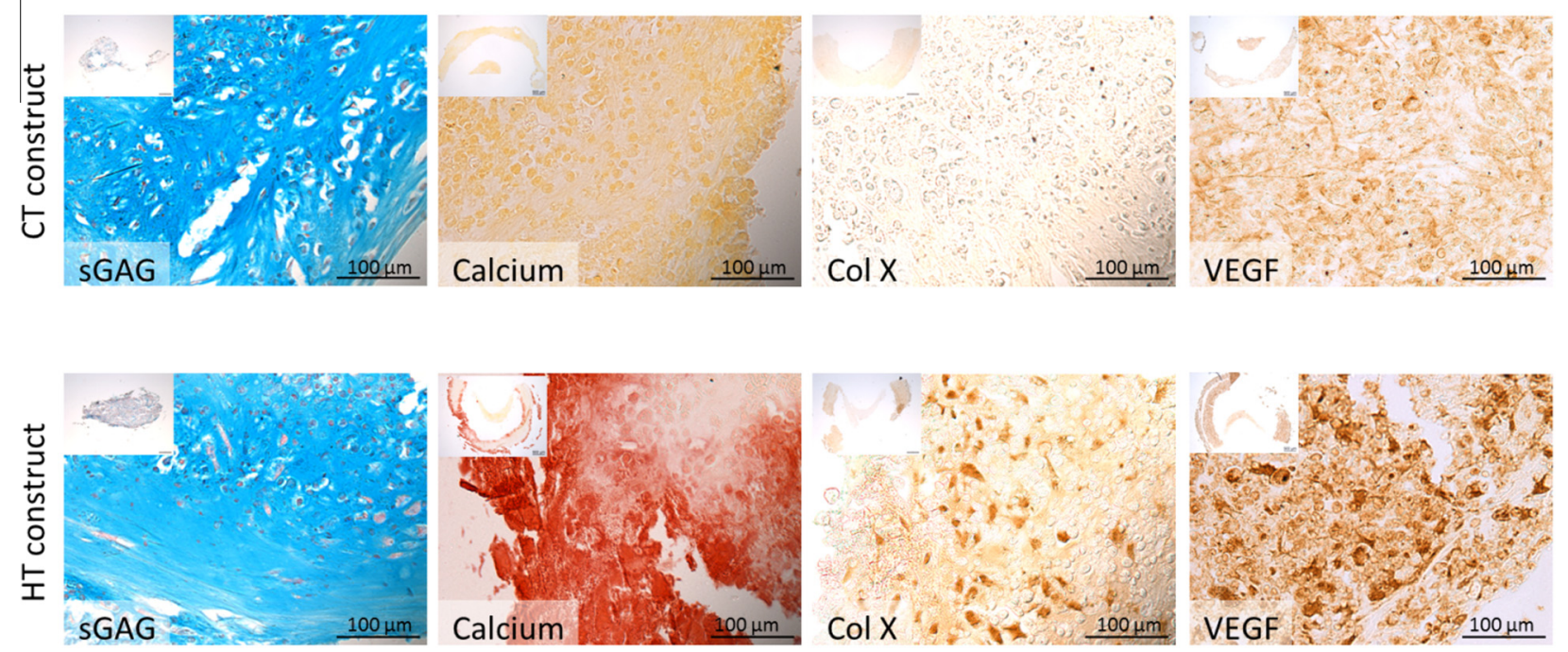

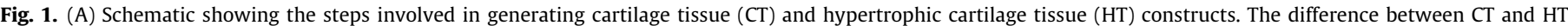

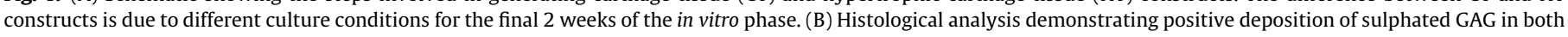

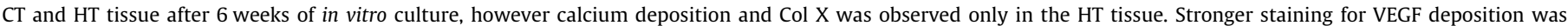
observed in HT constructs.

negative for calcification and saw only some peripheral type X collagen deposition, while late hypertrophic tissues stained positive for these markers of hypertrophy [12]. In addition, we found that VEGF accumulation was greater in engineered hypertrophic tissues compared to cartilaginous tissues. Differences in the levels of ECM-bound VEGF retained in decellularized scaffolds may explain, at least in part, the greater levels of vascularization observed within the HT derived scaffolds compared to the CT derived scaffolds upon subcutaneous implantation into nude mice. Furthermore, only scaffolds derived from devitalized HT tissue were able to support ectopic mineralization and immature bone formation. An explanation for this may be that by facilitating vascularization, a key requirement for osteogenesis, these HT constructs are able to support host-mediated ectopic bone formation through the ingrowth of vessels and subsequent delivery of progenitor cells and growth factors. The presence of mineral within the HT scaffolds may also support osteogenesis [37-39]. In addition, it is likely that the hypertrophic culture conditions are promoting the differential expression and production of growth factors that are retained within the HT scaffolds and which may be facilitating osteogenesis. Future studies are required to quantify the broad range of pro-angiogenic and pro-osteogenic growth factors believed to be present in engineered hypertrophic cartilage.

The tissue that formed within the HT scaffolds following subcutaneous implantation stained positively for sGAG and type X collagen deposition, suggesting that a sub-population of the progenitor cells that are recruited into these scaffolds may be undergoing chondrogenesis and generating bone via the process of endochondral ossification. Indeed, other studies have demonstrated that ectopic bone formation within growth factor delivery scaffolds also occurs through this endochondral pathway [40]. In contrast, recent studies have suggested that bone formation induced by devitalized cartilaginous ECM cannot be defined as being of the endochondral origin [21]. Further work is required to track the fate of progenitor cells that are recruited into devitalized ECM derived scaffolds in vivo.

Interestingly, the viable $\mathrm{CT}$ and $\mathrm{HT}$ grafts demonstrated a comparable capacity to promote ectopic bone formation, suggesting that in vivo both engineered tissues progress along a similar endochondral pathway. Both viable tissues formed ring-shaped constructs via self-assembly during in vitro culture, and these shapes were maintained following in vivo implantation. It should be noted that the degree of bone formation within the scaffolds derived from devitalized engineered ECM was inferior to that generated by these viable engineered tissues, confirming that chondrogenically primed MSCs within a cartilage graft play an important role in driving osteogenesis $[41,42]$. A recent study also compared the efficacy of decellularized native cartilage based scaffolds, implanted subcutaneously either cell-free or seeded with MSCs, to promote endochondral bone formation [22]. That study found increased levels of mineralization within the cell-seeded scaffolds, further confirming the key role played by viable donor cells in driving osteogenesis in these models. Devitalized scaffolds generated from cell-derived ECM would however appear to retain key structural and functional molecules that support angiogenesis and bone formation. ECM from various sources have been studied previously 
(A)

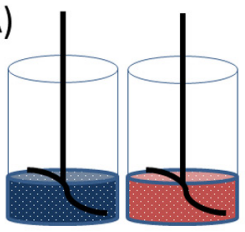

Constructs blended to form a

homogenous slurry

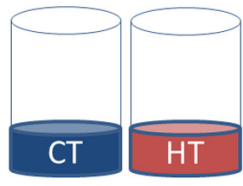

Slurry treated to decellularise- left with cell-derived matrix only

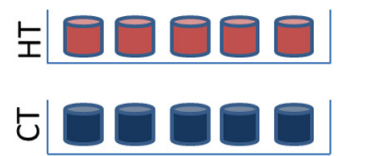

Slurry placed in moulds and freeze-dried using optimised protocol

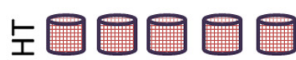

七圆田圆园

Porous scaffolds containing only cell-derived matrix components
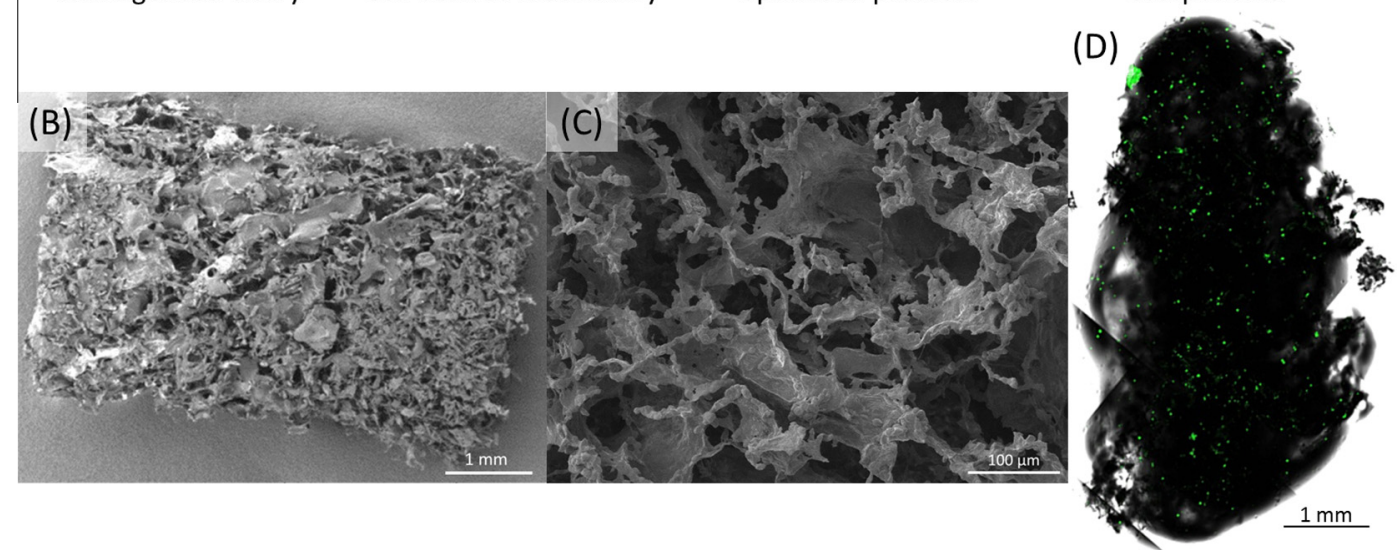

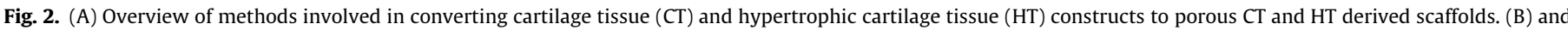

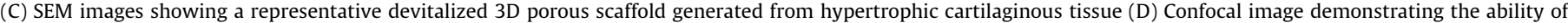
seeded MSCs to infiltrate into the centre of HT derived scaffolds within $24 \mathrm{~h}$ in vitro.

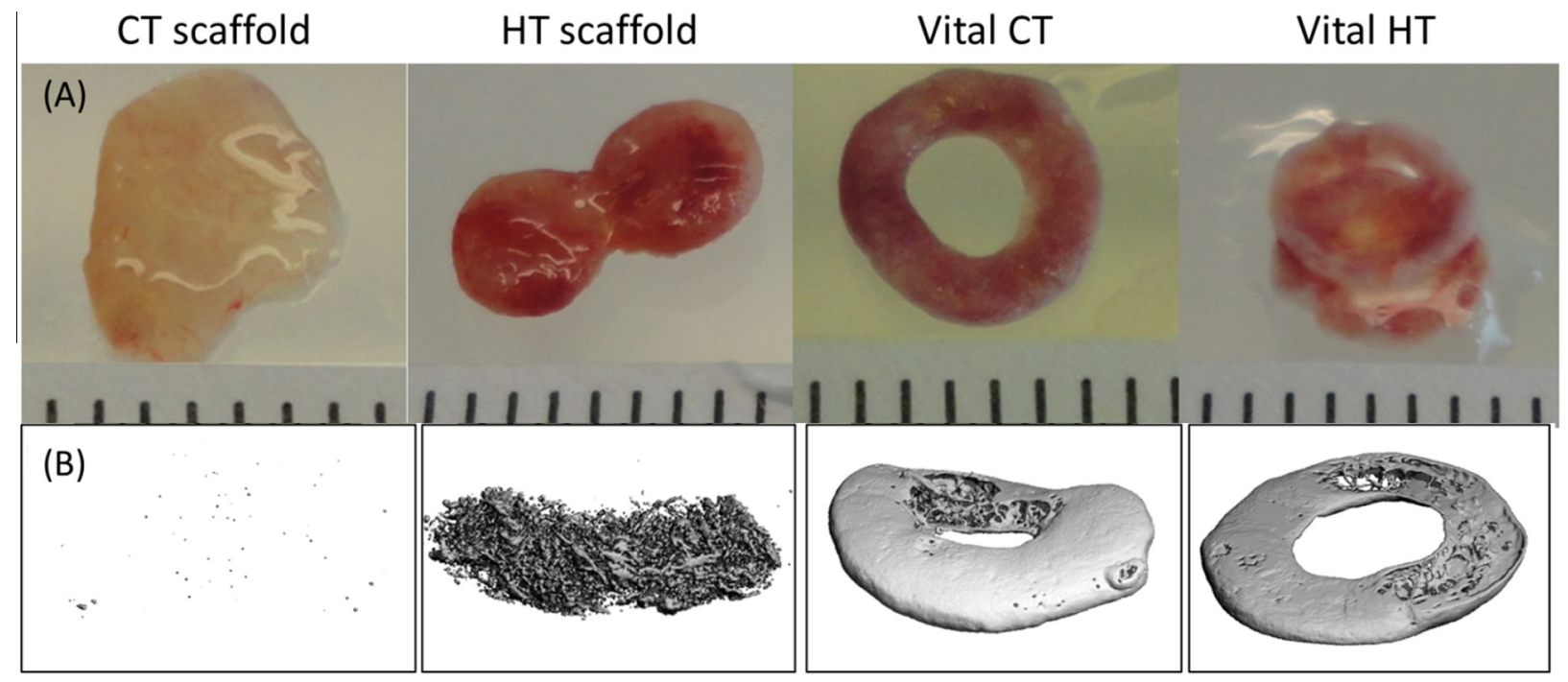

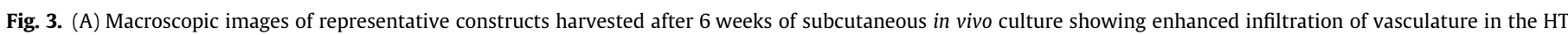

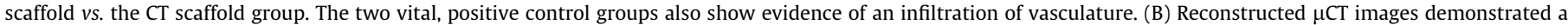
significantly greater level of mineral deposition in the HT scaffold vs. the CT scaffold.

as biomaterials for tissue regeneration, as ECM has a number of obvious advantages for tissue engineering applications $[17,35,43,44]$. The data presented in this study add to the body of work demonstrating the utility of different cell and native tissue-derived ECM to be used as scaffolds in orthopaedic medicine $[21,25,45-52]$.

HT derived scaffolds were also found to promote bone regeneration in critically-sized defects in a rat femoral defect study. It should be noted that some host-to-host variability in the extent of scaffold-mediated bone repair was observed. The scaffolds were manufactured consistently from one batch of slurry, and so variability is thought to be due primarily to host-to-host differences, or possibly due to slight procedural differences during the surgeries, although no healing was observed in any of the empty defect controls. For example, the periosteum was removed during surgery, and as a rich source of progenitor cells it is possible that varying amounts of residual periosteum had a differential effect on healing. Future studies will address this issue of host-to-host variability through the use of larger sample sizes, and will include additional control groups such as cell-free collagen based scaffolds 


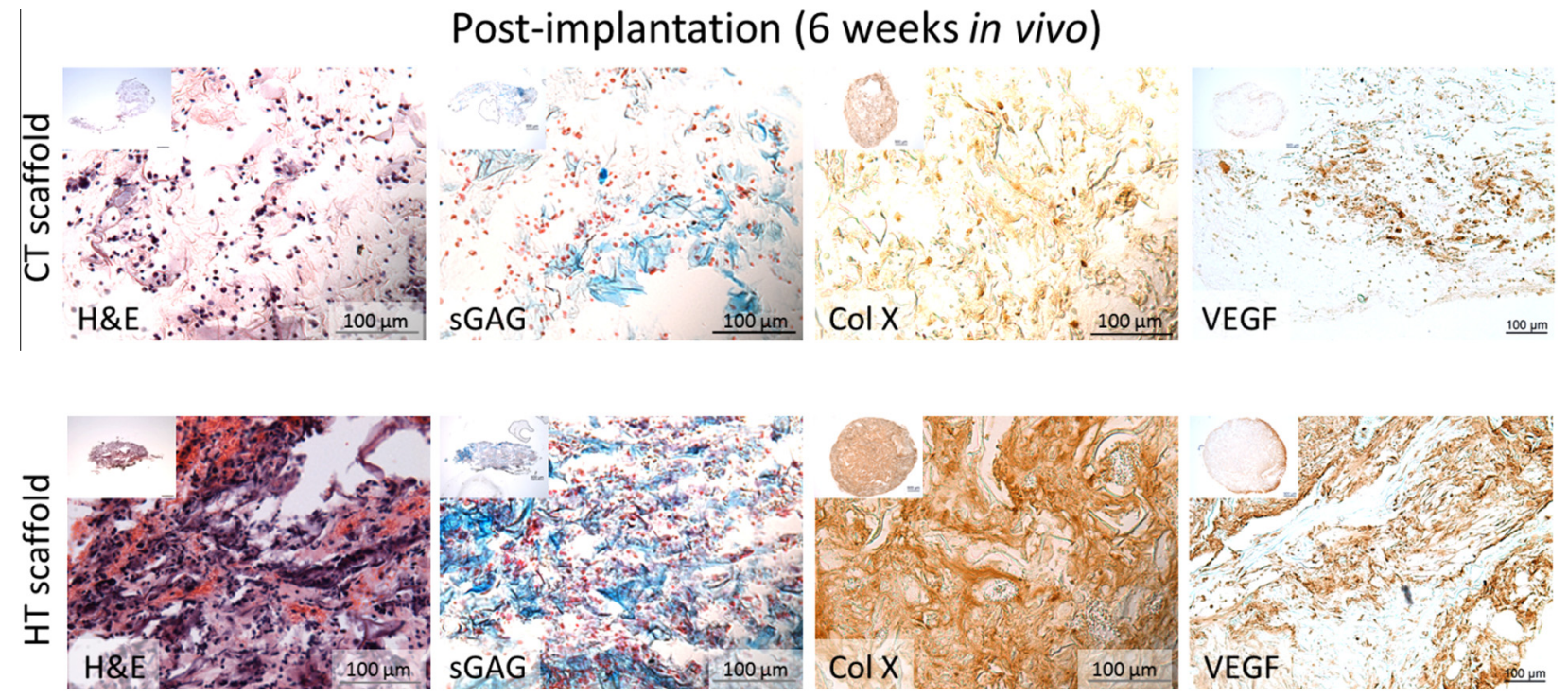

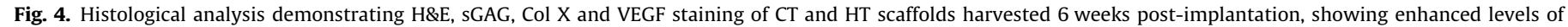
staining in the HT scaffold group.

(A)

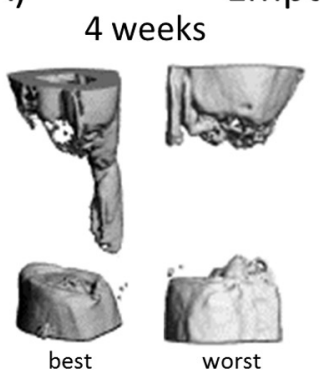

(C)

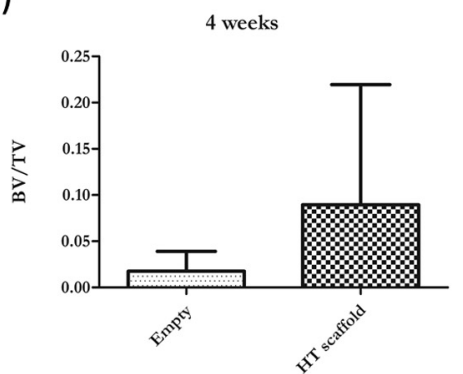

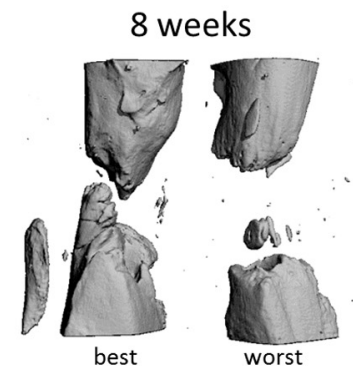

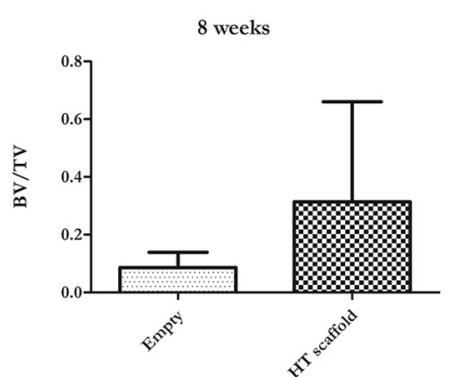

(B)

4 weeks

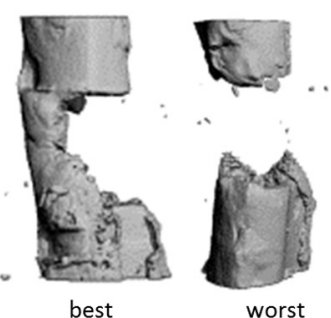

(D)
HT scaffold

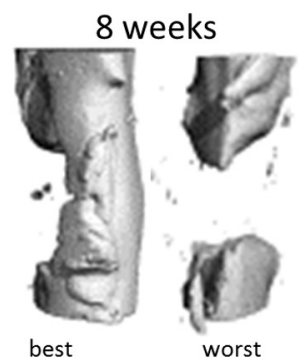

BV/TV vs. Time

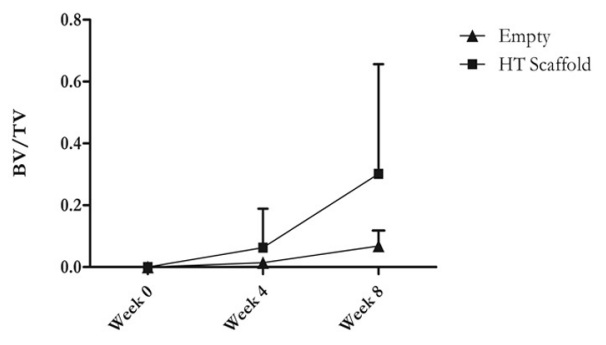

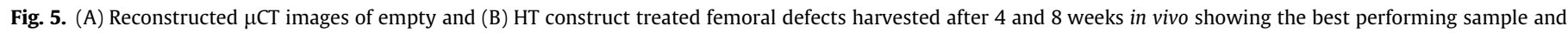

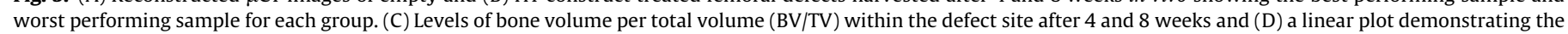
increase in BV/TV over time in both empty and HT construct treated defects.

soaked in hypertrophic culture media (as elements in this media such as thyroxine may help stimulate long bone repair).

In conclusion, numerous studies have demonstrated the ability of viable chondrogenic cells to induce bone formation in both ectopic and orthotopic environments; however many scientific, logistical and regulatory concerns remain about expanding these methods to generate grafts for clinical use. Clinical use of acellular ECM derived scaffolds is a strategy that could be easier to translate than tissue engineering viable cell-based constructs, and previous successful products have shown the feasibility of commercializing decellularized ECM materials with FDA approval [18]. Key advantages of this strategy include off-the-shelf availability, as well as the ability to create scaffolds of different complex sizes. To the best of our knowledge, this study is the first to investigate the use of a decellularized engineered hypertrophic cartilage matrix as a scaffold for orthotopic bone repair, demonstrating the potential of this approach. It builds on a body of work demonstrating the use of devitalized engineered ECM for tissue regeneration, including a recent study using death inducible MSCs to deposit a hypertrophic cartilage ECM that was subsequently shown to promote ectopic bone formation [21]. With all of these strategies, including that proposed in this study, scaling-up the manufacture of engineered tissues will be a significant challenge, and will be hampered by the difficulties involved with culturing the very large numbers of 

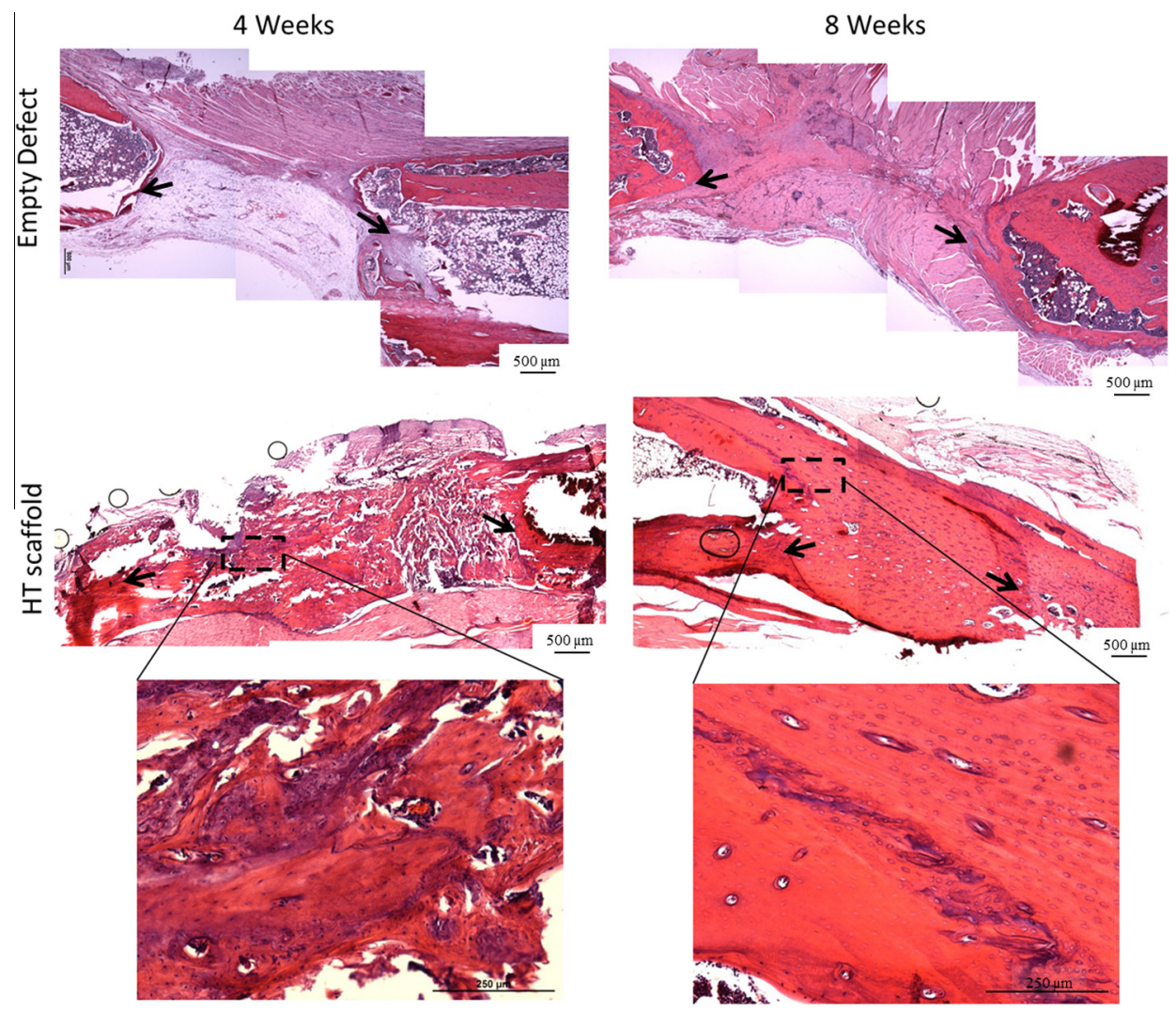

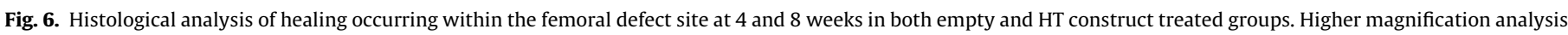
demonstrates evidence of lamellar bone evident within fully-bridged HT-treated defects 8 weeks post-implantation.

cells required to engineer cartilaginous grafts. Therefore it may be possible that naturally derived hypertrophic cartilage from native tissue (e.g. the growth plate) could provide an alternative source of scaffolding material. Future studies from our lab will continue to explore the use of both decellularized native and engineered cartilaginous tissues for bone regeneration.

\section{Conclusion}

Taken together, these results show the potential of using hypertrophic cartilage matrix as a scaffold material for the repair of large bone defects. Host cells were observed to migrate throughout the construct and deposit de novo bone matrix ectopically and in a critically-sized defect model. Such a scaffold could potentially be combined with a patient's cells during surgery, and/or additional growth factors or biological cues, to further accelerate the healing process.

\section{Acknowledgements}

This work was funded by the AO Foundation under the large bone defect healing program. Surgical advice, PEEK fixator plates and custom designed surgical instruments were kindly provided by Professor Steve Goldstein, University of Michigan. Human bone marrow-derived MSCs were kindly provided by REMEDI, National Centre for Biomedical Engineering Science, National University of Ireland Galway, Ireland and funded by Science Foundation Ireland (Grant Number 09/SRC/B1794). This work was also funded by the Health Research Board of Ireland (HRA_POR/2011/27) and the European Research Council (ERC grant agreement no. 239685).

\section{Appendix A. Figures with essential colour discrimination}

Certain figures in this article, particularly Figs. 1-4, and 6, are difficult to interpret in black and white. The full colour images can be found in the on-line version, at http://dx.doi.org/10.1016/ j.actbio.2015.05.031.

\section{References}

[1] F.G. Lyons, A.A. Al-Munajied, S.M. Kieran, M.E. Toner, C.M. Murphy, G.P. Duffy, et al., The healing of bony defects by cell-free collagen-based scaffolds compared to stem cell-seeded tissue engineered constructs, Biomaterials 31 (2010) 9232-9243.

[2] H.M. Kronenberg, Developmental regulation of the growth plate, Nature 423 (2003) 332-336.

[3] J. Dai, A.B.M. Rabie, VEGF: an essential mediator of both angiogenesis and endochondral ossification, J. Dent. Res. 86 (2007) 937-950.

[4] E. Farrell, O.P. Van Der Jagt, W. Koevoet, N. Kops, C.J. Van Manen, C.A. Hellingman, et al., Chondrogenic priming of human bone marrow stromal cells: a better route to bone repair?, Tissue Eng Part C Methods 15 (2009) 285295.

[5] E.J. Mackie, Y.A. Ahmed, L. Tatarczuch, K.S. Chen, M. Mirams, Endochondral ossification: how cartilage is converted into bone in the developing skeleton, Int. J. Biochem. Cell B 40 (2008) 46-62.

[6] D. Gawlitta, E. Farrell, J. Malda, L.B. Creemers, J. Alblas, W.J.A. Dhert, Modulating endochondral ossification of multipotent stromal cells for bone regeneration, Tissue Eng. Part B Rev. 16 (2010) 385-395.

[7] C.S. Bahney, D.P. Hu, A.J. Taylor, F. Ferro, H.M. Britz, B. Hallgrimsson, et al., Stem cell-derived endochondral cartilage stimulates bone healing by tissue transformation, J. Bone Miner. Res. 29 (2014) 1269-1282.

[8] N. Harada, Y. Watanabe, K. Sato, S. Abe, K. Yamanaka, Y. Sakai, et al., Bone regeneration in a massive rat femur defect through endochondral ossification achieved with chondrogenically differentiated MSCs in a degradable scaffold, Biomaterials 35 (2014) 7800-7810.

[9] J.I. Huang, M.M. Durbhakula, P. Angele, B. Johnstone, J.U. Yoo, Lunate arthroplasty with autologous mesenchymal stem cells in a rabbit model, J. Bone Joint Surg. 88 (2006) 744-752. 
[10] A. Muraglia, A. Corsi, M. Riminucci, M. Mastrogiacomo, R. Cancedda, P. Bianco, et al., Formation of a chondro-osseous rudiment in micromass cultures of human bone-marrow stromal cells, J. Cell Sci. 116 (2003) 2949-2955.

[11] E.J. Sheehy, T. Vinardell, C.T. Buckley, D.J. Kelly, Engineering osteochondral constructs through spatial regulation of endochondral ossification, Acta Biomater. 9 (2013) 5484-5492.

[12] C. Scotti, B. Tonnarelli, A. Papadimitropoulos, A. Scherberich, S. Schaeren, A Schauerte, et al., Recapitulation of endochondral bone formation using human adult mesenchymal stem cells as a paradigm for developmental engineering, Proc. Natl. Acad. Sci. USA 107 (2010) 7251-7256.

[13] J.M. Jukes, S.K. Both, A. Leusink, L.M.T. Sterk, C.A. Van Blitterswijk, J. De Boer, Endochondral bone tissue engineering using embryonic stem cells, Proc. Natl. Acad. Sci. USA 105 (2008) 6840-6845.

[14] P. Janicki, P. Kasten, K. Kleinschmidt, R. Luginbuehl, W. Richter, Chondrogenic pre-induction of human mesenchymal stem cells on $\beta$-TCP: Enhanced bone quality by endochondral heterotopic bone formation, Acta Biomater. 6 (2010) 3292-3301.

[15] E. Farrell, S.K. Both, K.I. Odörfer, W. Koevoet, N. Kops, F.J. O’Brien, R.J.B. De Jong, J.A. Verhaar, V. Cuijpers, J. Jansen, R.G. Erben, G.J.V.M. Van Osch, In-vivo generation of bone via endochondral ossification by in-vitro chondrogenic priming of adult human and rat mesenchymal stem cells, BMC Musculoskelet. Disord. 12 (2011)

[16] H.A. Awad, R.J. O'Keefe, C.H. Lee, J.J. Mao, Bone Tissue Engineering: Clinical Challenges and Emergent Advances and Prospects in Orthopaedic and Craniofacial Surgery, Elsevier/Academic Press, 2012.

[17] K.E.M. Benders, P.R.v. Weeren, S.F. Badylak, D.B.F. Saris, W.J.A. Dhert, J. Malda, Extracellular matrix scaffolds for cartilage and bone regeneration, Trends Biotechnol. 31 (2013) 169-176.

[18] C.W. Cheng, L.D. Solorio, E. Alsberg, Decellularized tissue and cell-derived extracellular matrices as scaffolds for orthopaedic tissue engineering, Biotechnol. Adv. 32 (2014) 462-484.

[19] F. Moroni, T. Mirabella, Decellularized matrices for cardiovascular tissue engineering, Am. J. Stem Cells 3 (2014) 1-20.

[20] P.M. Crapo, T.W. Gilbert, S.F. Badylak, An overview of tissue and whole organ decellularization processes, Biomaterials 32 (2011) 3233-3243.

[21] P.E. Bourgine, C. Scotti, S. Pigeot, L.A. Tchang, A. Todorov, I. Martin, Osteoinductivity of engineered cartilaginous templates devitalized by inducible apoptosis, Proc. Natl. Acad. Sci. 111 (2014) 17426-17431.

[22] D. Gawlitta, K.E.M. Benders, J. Visser, A.S. van der Sar, D.H.R. Kempen, L.F.H. Theyse, J. Malda, W.J.A. Dhert, Decellularized cartilage-derived matrix as substrate for endochondral bone regeneration, Tissue Eng. Part A 21 (2014) 694-703.

[23] A.J. Sutherland, G.L. Converse, R.A. Hopkins, M.S. Detamore, The bioactivity of cartilage extracellular matrix in articular cartilage regeneration, Adv. Healthc. Mater. 4 (2015) 29-39.

[24] C.Z. Jin, S.R. Park, B.H. Choi, K. Park, B.-H. Min, In vivo cartilage tissue engineering using a cell-derived extracellular matrix scaffold, Artif. Organs 31 (2007) 183-192.

[25] H.V. Almeida, Y. Liu, G.M. Cunniffe, K.J. Mulhall, A. Matsiko, C.T. Buckley, F.J. O'Brien, D.J. Kelly, Controlled release of transforming growth factor- $\beta 3$ from cartilage-extra-cellular-matrix-derived scaffolds to promote chondrogenesis of human-joint-tissue-derived stem cells, Acta Biomater. 10 (2014) 44004409.

[26] E. Kheir, T. Stapleton, D. Shaw, Z. Jin, J. Fisher, E. Ingham, Development and characterization of an acellular porcine cartilage bone matrix for use in tissue engineering, J. Biomed. Mater. Res. A 99A (2011) 283-294.

[27] T.T. Lau, L.Q.P. Lee, B.N. Vo, K. Su, D.-A. Wang, Inducing ossification in an engineered 3D scaffold-free living cartilage template, Biomaterials 33 (2012) 8406-8417.

[28] H. Lu, T. Hoshiba, N. Kawazoe, G. Chen, Autologous extracellular matrix scaffolds for tissue engineering. Biomaterials 32 (2011) 2489-2499.

[29] T. Mesallati, C.T. Buckley, D.J. Kelly, A comparison of self-assembly and hydrogel encapsulation as a means to engineer functional cartilaginous grafts using culture expanded chondrocytes, Tissue Eng. Part C Methods 20 (2014) 52-63.

[30] A.D. Murdoch, L.M. Grady, M.P. Ablett, T. Katopodi, R.S. Meadows, T.E. Hardingham, Chondrogenic differentiation of human bone marrow stem cells in transwell cultures: generation of scaffold-free cartilage, Stem Cells 25 (2007) 2786-2796.

[31] M.G. Haugh, C.M. Murphy, R.C. McKiernan, C. Altenbuchner, F.J. O’Brien, Crosslinking and mechanical properties significantly influence cell attachment, proliferation, and migration within collagen glycosaminoglycan scaffolds, Tissue Eng. Part A 17 (2010) 1201-1208.

[32] T. Vinardell, E.J. Sheehy, C.T. Buckley, D.J. Kelly, A comparison of the functionality and in vivo phenotypic stability of cartilaginous tissues engineered from different stem cell sources, Tissue Eng. Part A 18 (2012) $1161-1170$.

[33] T.M. Filion, X. Li, A. Mason-Savas, J.M. Kreider, S.A. Goldstein, D.C. Ayers, et al., Elastomeric osteoconductive synthetic scaffolds with acquired osteoinductivity expedite the repair of critical femoral defects in rats, Tissue Eng. Part A 17 (2011) 503-511.

[34] D.M. Faulk, S.A. Johnson, S.F. Badylak, Cardiac Regeneration and Repair, Woodhead Publishing Ltd., 2014. pp. 180-200.

[35] T.W. Gilbert, T.L. Sellaro, S.F. Badylak, Decellularization of tissues and organs, Biomaterials 27 (2006) 3675-3683.

[36] P.E. Bourgine, B.E. Pippenger, A. Todorov Jr, L. Tchang, I. Martin, Tissue decellularization by activation of programmed cell death, Biomaterials 34 (2013) 6099-6108.

[37] W.L. Murphy, C.A. Simmons, D. Kaigler, D.J. Mooney, Bone regeneration via a mineral substrate and induced angiogenesis, J. Dent. Res. 83 (2004) 204-210.

[38] A.A. Al-Munajjed, N.A. Plunkett, J.P. Gleeson, T. Weber, C. Jungreuthmayer, T. Levingstone, J. Hammer, F.J. O'Brien, Development of a biomimetic collagenhydroxyapatite scaffold for bone tissue engineering using a SBF immersion technique, J. Biomed. Mater. Res. B 90B (2009) 584-591.

[39] J.P. Gleeson, N.A. Plunkett, F.J. O'Brien, Addition of hydroxyapatite improves stiffness, interconnectivity and osteogenic potential of a highly porous collagen-based scaffold for bone tissue regeneration, Eur. Cell Mater. 20 (2010) 218-230.

[40] C.A. Simmons, E. Alsberg, S. Hsiong, W.J. Kim, D.J. Mooney, Dual growth factor delivery and controlled scaffold degradation enhance in vivo bone formation by transplanted bone marrow stromal cells, Bone 35 (2004) 562-569.

[41] M. Serafini, B. Sacchetti, A. Pievani, D. Redaelli, C. Remoli, A. Biondi, M. Riminucci, P. Bianco, Establishment of bone marrow and hematopoietic niches in vivo by reversion of chondrocyte differentiation of human bone marrow stromal cells, Stem Cell Res. 12 (2014) 659-672.

[42] C. Scotti, E. Piccinini, H. Takizawa, A. Todorov, P. Bourgine, A. Papadimitropoulos, A. Barbero, M.G. Manz, I. Martin, Engineering of a functional bone organ through endochondral ossification, Proc. Natl. Acad. Sci. USA 110 (2013) 3997-4002.

[43] J.E. Reing, L. Zhang, J. Myers-Irvin, K.E. Cordero, D.O. Freytes, E. Heber-Katz, K. Bedelbaeva, D. McIntosh, A. Dewilde, S.J. Braunhut, S.F. Badylak, Degradation products of extracellular matrix affect cell migration and proliferation, Tissue Eng. Part A 15 (2008) 605-614.

[44] R.A. Thibault, A.G. Mikos, F.K. Kasper, Scaffold/extracellular matrix hybrid constructs for bone-tissue engineering, Adv. Healthc. Mater. 2 (2013) 13-24.

[45] Q. Yang, J. Peng, Q. Guo, J. Huang, L. Zhang, J. Yao, F. Yang, S. Wang, W. Xu, A. Wang, S. Lu, A cartilage ECM-derived 3-D porous acellular matrix scaffold for in vivo cartilage tissue engineering with PKH26-labeled chondrogenic bone marrow-derived mesenchymal stem cells, Biomaterials 29 (2008) 2378-2387.

[46] N.C. Cheng, B.T. Estes, H.A. Awad, F. Guilak, Chondrogenic differentiation of adipose-derived adult stem cells by a porous scaffold derived from native articular cartilage extracellular matrix, Tissue Eng. Part A 15 (2009) 231-241.

[47] B.H. Choi, K.-H. Choi, H.S. Lee, B.R. Song, S.R. Park, J.W. Yang, B.-H. Min, Inhibition of blood vessel formation by a chondrocyte-derived extracellular matrix, Biomaterials 35 (2014) 5711-5720.

[48] K.-H. Choi, B.H. Choi, S.R. Park, B.J. Kim, B.-H. Min, The chondrogenic differentiation of mesenchymal stem cells on an extracellular matrix scaffold derived from porcine chondrocytes, Biomaterials 31 (2010) 5355-5365.

[49] Q. Yang, J. Peng, S.B. Lu, Q.Y. Guo, B. Zhao, L. Zhang, A.Y. Wang, W.J. Xu, Q. Xia, X.L. Ma, Y.C. Hu, B.S. Xu, Evaluation of an extracellular matrix-derived acellular biphasic scaffold/cell construct in the repair of a large articular high-loadbearing osteochondral defect in a canine model, Chin. Med. J. (Engl) 124 (2011) 3930-3938.

[50] E. Gruskin, B.A. Doll, F.W. Futrell, J.P. Schmitz, J.O. Hollinger, Demineralized bone matrix in bone repair: history and use, Adv. Drug Deliver. Rev. 64 (2012) 1063-1077.

[51] S. Zeitouni, U. Krause, B.H. Clough, H. Halderman, A. Falster, D.T. Blalock, C.D. Chaput, H.W. Sampson, C.A. Gregory, Human mesenchymal stem cell-derived matrices for enhanced osteoregeneration, Sci Transl Med 4 (2012) 132ra155.

[52] F. Pati, T.H. Song, G. Rijal, J. Jang, S.W. Kim, D.W. Cho, Ornamenting 3D printed scaffolds with cell-laid extracellular matrix for bone tissue regeneration, Biomaterials 37 (2015) 230-241. 